\title{
A (new) Phenomenon in Pulsar Dynamic Spectra
}

\author{
D. R. Stinebring, K. M. Becker, J. E. Espinoza Goodman, M. A. \\ Kramer, J. L. Sheckard \\ Dept. of Physics, Oberlin College, Oberlin, OH 44074
}

J. M. Cordes

Astronomy Dept., Cornell Univ., Ithaca, NY 14853-6801

T. J. Lazio

NRL, Code 7210, Washington, DC, 20375-5351

\begin{abstract}
Several studies in the last decade have explored dramatic instances of refractive fringing in dynamic spectra. We draw attention to a subtler but more widespread refractive effect: wisp-like structures extending (either linearly or with curvature) outward from the origin in the secondary spectrum plane. We have employed a simple pinhole screen simulation to investigate this phenomenon. It appears that the presence of one or more wisps in a secondary spectrum indicates a clump of scattering material that is significantly offset from the optical axis. Unlike the dramatic fringing events that have been investigated in the past, however, the interference that gives rise to a wisp is interference between rays within a scattering clump rather than between two major ray bundles. Furthermore, the scattering clump must be significantly elongated along the direction of pulsar motion. The range of fringe spacings that this produces, all with a similar slope in the secondary spectrum, gives rise to the linear extent of the wisp in the secondary spectrum.
\end{abstract}

Refraction has a strong influence on pulsar dynamic spectra. The bending of wavefronts by $\mathrm{AU}$-sized structures in the interstellar medium causes tilting of scintles in the frequency-time plane and the presence of quasi-periodic fringing patterns across dynamic spectra. The frequent presence of refraction in dynamic spectra presents a problem since a Kolmogorov density inhomogeneity spectrum - favored by many other observations - would infrequently give rise to such effects.

We focus here on wisp-like features that extend outward from the origin of the secondary spectrum plane. In observations we made at Arecibo in 1999 January, we found occasions in which the wisp-like features in the secondary spectrum of PSR $1133+16$ were remarkably sharp and well-defined, albeit appearing at a low power level (about $30 \mathrm{~dB}$ below the main diffraction peak). Other studies (Cordes \& Wolszczan 1986; Wolszczan \& Cordes 1987; Rickett, Lyne, \& Gupta) show examples of this phenomenon, which is often seen in dynamic spectra as interference fringes with nearly constant position angle but 
a large range of spacings, similar in appearance to the ridge lines of a heavily folded mountain range.

We have used the simulation to explore what kind of scattering scenario could cause these wisps. A single screen is placed along the line of sight between the pulsar and the observer. The screen is perforated with pinholes that define discrete ray paths between the pulsar and the observer. The pinholes can be organized into clumps that approximate "subimages" on the sky. The interference between all the ray paths is calculated at a point in the observers plane, with time development provided by pulsar motion transverse to the line of sight (oriented along the $\mathrm{x}$-axis). The interference calculation is performed over a range of wavelengths in order to calculate the frequency dimension of the dynamic spectrum. An adjustable amount of noise is added to the dynamic spectrum to make it more realistic and to suppress the sidelobes that arise from the interference between pairs of rays.

A single pair of pinholes produces a two-dimensional sinusoidal pattern in the dynamic spectrum. This sinusoidal component has coordinates $n_{f}$ and $n_{t}$ in the power spectrum of the dynamic spectrum (the so-called secondary spectrum) given by

$$
n_{f}=\left(\theta_{1}^{2}-\theta_{2}^{2}\right) \frac{L}{2 c} \quad n_{t}=\frac{\vec{V} \cdot\left(\overrightarrow{\theta_{1}}-\overrightarrow{\theta_{2}}\right)}{\lambda},
$$

where $L$ is the distance from the observer to the screen, $\lambda$ is the observing wavelength, $\overrightarrow{\theta_{1,2}}$ are the vector positions of the rays, and $\vec{V}$ is the velocity vector (all vectors are two-dimensional, transverse to the line of sight).

Points lying on the same side of the optical axis will all have the same sign of $\vec{V} \cdot\left(\overrightarrow{\theta_{1}}-\overrightarrow{\theta_{2}}\right)$ and $\left(\theta_{1}^{2}-\theta_{2}^{2}\right)$. Hence, the ratio $n_{t} / n_{f}$, which is the slope in the secondary spectrum, will have a consistent sign for intra-clump interference if all points lie on the same side of the optical axis. Furthermore, if the angular extent of the clump is small compared to the mean offset of the clump, the value of this slope will have nearly the same value for all the interfering pairs in the clump.

Following this approach, we have been able to construct clumps of offset pinholes that approximate the wisps we see in our Arecibo data. Although we recognize that this solution is not unique, we find it suggestive that such a simple model produces this relatively widespread phenomenon. Furthermore, we note that since it is the intra-clump interference that causes the wisps, they may be present when the inter-clump interference terms are absent. This is possible because the inter-clump interference terms, which typically produce an "island" of power in the secondary spectra, will, if the clumps are too far apart, produce a fringe pattern that varies too rapidly in frequency or time to be resolved by the observations. Hence, that will leave the wisp features as the sole indicators that the source is split up into several linearly elongated subimages.

\section{References}

Cordes, J. M. \& Wolszczan, A. 1986, ApJ, 307, L27

Rickett, B. J., Lyne, A. \& Gupta, Y. 1997, MNRAS, 287, 739

Wolszczan, A. \& Cordes, J. M. 1987, ApJ, 320, L35 